\title{
Assessment of in vivo Bone Microarchitecture Changes in a Psoriatic Arthritic Patient Resulting from an Anti-TNFa Treatment
}

Enrico Soldati ( $\nabla$ enrico.soldati@univ-amu.fr)

Aix-Marseille University https://orcid.org/0000-0003-2818-7500

Lucas Escoffier

Aix-Marseille-University: Aix-Marseille Universite

Sophie Gabriel

Aix-Marseille-University: Aix-Marseille Universite

Jean Pierre Mattei

Aix-Marseille-University: Aix-Marseille Universite

Serge Cammilleri

Aix-Marseille-University: Aix-Marseille Universite

David Bendahan

Aix-Marseille-University: Aix-Marseille Universite

Sandrine Guis

Aix-Marseille-University: Aix-Marseille Universite

Research article

Keywords: bone microarchitecture, anti-TNFa, in vivo, psoriatic arthritic patient

Posted Date: December 1st, 2020

DOl: https://doi.org/10.21203/rs.3.rs-115381/v1

License: (9) (i) This work is licensed under a Creative Commons Attribution 4.0 International License.

Read Full License 


\section{Abstract}

Objective: Psoriatic arthritis (PA) is an inflammatory rheumatism, mediated in part by TNFa and associated with bone loss. Anti-TNFa treatment should inhibit this phenomenon and reduce the systemic bone loss. Ultra-high field MRI (UHF MRI) may be used to quantify bone microarchitecture (BM) in-vivo. In this study, we quantified BM using UHF MRI in a PA patient and followed up the changes related to antiTNFa treatment.

Patients and methods: A non-treated PA patient with knee arthritis and 7 gender-matched controls were scanned using a gradient re-echo sequence at UHF MRI. After a year of Adalimumab treatment, the patient underwent a second UHF MRI. A PET-FNa imaging was performed before and after treatment to identify and localize the abnormal metabolic areas. BM was characterized using typical morphological parameters quantified in 32 regions of interest (ROIs) located in the patella, proximal tibia, and distal femur. Statistical analysis was assessed performing Student T-tests.

Results: Before treatment, the BM parameters were statistically different from controls in 24/32 ROIs with differences reaching up to $38 \%$. After treatment, BM parameters were normalized for 15 out of $24 \mathrm{ROIs}$. The hypermetabolic areas disclosed by PET-FNa before the treatment partly resumed after the treatment.

Conclusion: Thanks to UHF MRI, we quantified in-vivo BM anomalies in a PA patient and we illustrated a major reversion after one year of treatment. Moreover, BM results highlighted that the abnormalities were not only localized in hypermetabolic regions identified by PET-FNa, suggesting that the bone loss was global and not related to inflammation.

\section{Introduction}

Psoriatic arthritis (PA) is an inflammatory rheumatism associated with psoriasis in which axial and peripheral joints can display an elevated inflammatory status [1]. PA has been initially described by Moll and Wright as a seronegative inflammatory arthritis that occurs most of the time in the presence of psoriasis [2]. It was initially thought to be rare but recent studies indicated that it might occur in up to $30 \%$ of patients with psoriasis [3], [4]. The main symptoms are asymmetric oligo-arthritis, polyarthritis, distal interphalangeal joint inflammation, dactylitis, low back pain and enthesis [1], [5]. Structural damage such as bone erosion and bone formation are frequently associated (11). Psoriasis and psoriatic arthritis are characterized by tissue infiltration by activated T cells thereby resulting in an increased TNFa, IL 17 and IL 23 production [5]-[8]. Synovial tissue and entheses are more particularly affected [9]. This pro inflammatory status can be an effective trigger of osteoclasts differentiation and activation through the expression of the receptor activator of nuclear factor kappa B ligand (RANKL)[10].

The systemic bone loss resulting in a reduced bone mineral density (BMD) and the role of TNFa antibodies in this process are a matter of debate in psoriatic arthritis [11]-[14][15]-[17]. So far, these changes have been assessed using dual energy $X$ ray absorptiometry (DXA), which is the gold standard for the diagnosis of osteoporosis [18]. In that respect, BMD values have been reported but bone micro 
architecture has never been documented as part of this bone alteration process. Interestingly, magnetic resonance imaging (MRI) and more particularly ultra-high field MRI (UHF MRI) has been reported as a promising tool for the assessment of bone microarchitecture given the high resolution of the corresponding images [19]. Over the last few years, this non-radiating imaging technique has shown promising results regarding the spine and femur trabeculation in osteoporosis [20]-[22]. So far, the corresponding changes in psoriatic arthritis have never been assessed.

The purpose of the present study was to investigate bone trabeculation in a patient with psoriatic arthritis using UHF MRI and to assess changes related to a TNFa antibodies therapeutic strategy.

\section{Material And Methods}

\section{Subject Recruitment}

This study had institutional review board approval and written informed consent was obtained from all the recruited subjects. One PA patient (male, 18 years old, body mass index $(\mathrm{BMI})=14.53 \mathrm{~kg} / \mathrm{m}^{2}$ ) was assessed before and after a one-year Adalimumab treatment. He was naïve of any conventional synthetic Disease Modifying Anti-Rheumatic Drug (CsDMARD), biological Disease Modifying Anti-Rheumatic Drug (bDMARD) or targeted synthetic Disease Modifying Anti-Rheumatic Drug (tsDMARD). Seven healthy volunteers with no sign of trabecular bone diseases or osteopenia (all males, mean age $=21.6$ years [interquartile range $(I Q R)=1$ year], mean $\left.B M I=21.32 \mathrm{~kg} / \mathrm{m}^{2}\left[\mathrm{IQR}=1.29 \mathrm{~kg} / \mathrm{m}^{2}\right]\right)$ were included in the control group.

\section{MRI scanning}

The patient and the volunteers underwent 7T MRI (MAGNETOM, Siemens Healthineers, Germany) of the knee articulation (distal femur, proximal tibia and patella). All subjects were scanned using a 28-channel knee coil and a 3D gradient recalled echo sequence (3D GRE, TR/TE $=15 / 4.36 \mathrm{~ms}$, flip angle $=12^{\circ}$, bandwidth $=326 \mathrm{~Hz} /$ pixel, field of view $=180 * 180 \mathrm{~mm}$, matrix $=768 \times 768$, in-plane voxel dimension $0.234 \times 0.234 \mathrm{~mm}$, slice thickness $=1.5 \mathrm{~mm}, 64$ sagittal planes, acquisition time $=5$ minutes 56 seconds). The PA patient was scanned once before treatment and once after one year of treatment. During MRI scanning, the patients' knee was immobilized by sandbags and secured by Velcro straps to avoid involuntary movements.

\section{PET-MRI image fusion}

MR and CT/PET FNa images [23] were acquired on two different scanners. Given that bones were clearly visible in both CT and MR images, the four bones (femur, tibia, fibula, and patella) were used as landmarks for the registration of both images. More specifically, bones were delineated semiautomatically in each stack of images and linear affine registrations were computed independently between each bone using FSL-FLIRT [24]. Each local affine transformation was then merged into a global 3D deformation field through the implementation described in [25] of the log-euclidean poly-affine 
framework proposed by Arsigny et al. [26]. The resulting deformation field was used to overlay the PET maps on the highly resolved and contrasted 7T MR anatomical images as previously reported [27] (Fig. 1).

\section{PET-MR Image Analysis}

Fused PET-MR images were visually evaluated by an expert (SG) with the aim of identifying and localizing the hypermetabolic regions before and after the treatment. The visual inspection of fused images was crucial in order to identify the regions with hyperintense signals.

Bone volume fraction maps representing the relative volume of bone within each voxel were generated from the GRE images. The initial images were linearly scaled in order to cover the range from 0 (pure bone) to 255 (pure marrow) [28], [29]. On each image, distal femur, proximal tibia and patella were delineated using the Chan-Vese algorithm [30]. The corresponding filled contours were used as masks on which a 10-pixels closing process was applied $(2.34 \mathrm{~mm})$ in all directions in order to eliminate the cortical bone (Fig. 2). Several region of interests (ROI) where identified in different locations of the trabecular bone in order to fully investigate the trabecular network.

\section{ROls selection:}

Patella: The first set of ROIs (ROI1, ROI1 a, ROI1b and ROI1c) were located in the patella region and referred respectively to the trabecular space of the whole patella, the upper and lower third of the trabecular region where the quadriceps and patellar tendons are respectively attached and the central third of the patella (Fig. 2).

Distal Femur: ROI2 was located in the distal femur epiphysis as illustrated in Fig. 2.

Proximal Tibia: The final set of ROIs ( $\mathrm{ROI} 3, \mathrm{ROI} 3 \mathrm{a}$ and $\mathrm{ROI} 3 \mathrm{~b}$ ) were positioned in the proximal tibia. $\mathrm{ROI} 3$ refers to the trabecular space of the proximal tibia epiphysis. ROI3a represents the trabecular part of the tibia where the medial collateral ligand is attached and ROI3b represents the trabecular part of the tibia where there was no hypermetabolic activity on the basis of the PET FNa signal. (Fig. 2).

These ROIs selection was based on the PET-FNa results. Accordingly they were selected in regions with hyper-intense signals before the Adalimumab treatment and were selected in the same regions after the treatment regardless of the signal intensity.

\section{Bone Microstructure Evaluation}

To reduce the computational costs from the 3D ROIs, three 2D centrally located planes were selected for each subject i.e. the image with the highest $\mathrm{ROI}$ surface together with the $\mathrm{N}_{+1}$ and $\mathrm{N}_{-1}$ images.

ROls were then binarized using an automatic local thresholding as previously described [31] and three independent metrics were computed. The bone volume fraction (BVF) which refers to the ratio between bone and the total volume, the trabecular thickness (Tb.Th) and spacing (Tb.Sp). Tb.Th and Tb.Sp were 
extrapolated using iMorph [32] which can generate an aperture map (AM) derived from a distance transformation map. The AM was retrieved from the maximal balls diameter enclosed in the bone (Tb.Th) and in the marrow (Tb.Sp) phases (Fig. 2). Finally the trabecular number (Tb.N) was computed as the ratio between the BVF and the Tb.Th.

Student's T-tests were used in order to assess the morphological parameters differences between the control group and the PA patient before and after the TNF treatment. For each subject, three measurements were obtained for each metric and each ROI. A p-value lower than 0.01 was considered as significant.

\section{Standardized Uptake Values}

A semi-quantitative analysis of PET images was performed as previously described in order to generate the Standardized Uptake Values (SUV) [23], [33]. SUV were computed as the ratio between the signal intensity within each pixel of the image scaled to the concentration of the total injected radioactivity ( $3 \mathrm{MBq} / \mathrm{Kg}$ ). The corresponding results refer the pixel-based metabolic. A SUV of 2.5 or higher is generally considered to be indicative of an "hypermetabolic" region. Finally, mean and maximal values were computed within each ROI.

\section{Results}

\section{PET-FNa: Hypermetabolism evolution}

The visual inspection of the initial PET image showed intense polyarticular hyperintense signals preferentially involving the knees, the left hip, the right ankle, the elbows, and more moderately the spine, the feet and the hands. As illustrated in Fig. 1, large hyperintensities were observed in the knee. The second PET image recorded after one year of treatment, showed an unequivocal reduction in most of the hypermetabolic regions affecting the joints of the axial and appendicular skeleton and more particularly the knee. The whole set of ROls showed reduced hyperintensities whereas no more hyperintense signal was visible for $\mathrm{ROI} 2$ and $\mathrm{ROI} 3 \mathrm{~b}$

\section{PET-FNa: SUV results}

SUV were quantified in all the knees ROIs before and after one year of treatment and the corresponding values are indicated in table 2 . Before the treatment, SUVmean was abnormal in 5 over 8 ROIs. The abnormal values were concentrated in all the patellar ROIs $(2.7 \pm 0.1)$ and ROI3a (2.8). SUVmax averaged over the whole set of ROIs was $3.67 \pm 0.41$. After the treatment, SUV were no longer larger than 2.5 in almost all the ROIs while the averaged SUVmax was also significantly reduced i.e. $2.86 \pm 0.86$. Large SUV values (i.e. between 1.7 and 2.5) were still visible in all the patella ROIs and ROI3a (Table 2).

MRI microarchitecture 
Regarding the MRI-based micro-architecture measurements performed before the treatment, the patient was outside the control range for multiple metrics and multiple localizations (24 out of 32 measurements were statistically different from the controls). However, after one year of treatment the microarchitectural parameters differences between the PA patient and the healthy references were reduced and the parameters were approaching or within the control range (only 9 out of 32 measurements were still statistically different than controls) (table 2).

\section{Patella}

Before the treatment and considering the four ROls delineated in the patellar region, BVF of the patient was always significantly lower as compared to controls with a mean difference of $32 \pm 19 \%$. The Tb.Th difference was always below $5 \%$ ( $p>0.01$ for all the four ROls), with a general mean of $0.245 \pm 0.010 \mathrm{~mm}$ for the controls and $0.244 \pm 0.012 \mathrm{~mm}$ for the patient. The Tb.Sp difference was statistically significant for ROI1, ROI1b and ROI1 c but not for ROI1a with the patient having larger trabecular spaces as compared to controls and therefore a negative difference mean of $-32 \pm 8 \%$. Similar results were found for Tb.N and a significant difference was found for ROI1, ROI1b and ROI1 c but not for ROI1 a with a general mean difference of $27 \pm 8 \%$. Following the 12-month of TNF treatment, most of the micro-architecture metrics but Tb.Th reversed to normal values. BVF increased in the four patella's ROls thereby reducing the differences with controls to a non-significant mean value of $2 \pm 5 \%$. Similar results were quantified for Tb.Sp and Tb.N with a non-significant difference with controls for any of the patella's ROls and a new overall patient mean difference of $-9 \pm 6 \%$ for Tb.Sp and $10 \pm 9 \%$ for Tb.N. On the contrary, after the treatment, Tb.Th became significantly larger with a significant difference (up to $8 \%$ ) with controls and so for ROI1, ROI1b and ROI1c (Fig. 3 and table 2).

\section{Distal Femur}

In the distal femur (ROI2) the difference between the healthy reference and the patient before the treatment was more than $30 \%$ for all the parameters (38\% for BVF, $-33 \%$ for Tb.Sp and $32 \%$ for Tb.N) except for Tb.Th for which the diffrence was less than $1 \%$.

The image analysis after the treatment still showed increased BVF and Tb.N values for, and reduced Tb.Sp values. The corresponding differences between the patient and the control values were $13 \%, 14 \%$ and $-21 \%$ respectively. Similar to the results found in the patella, the Tb.Th increased becoming $3 \%$ thicker than controls. The difference between the control and the patient values after the treatment was statistically significant $(p>0.01)$ for none of the micro-architectural parameters evaluated (table 2$)$.

\section{Proximal Tibia}

The three ROIs ( $\mathrm{ROI} 3, \mathrm{ROI} 3 \mathrm{a}$ and $\mathrm{ROI} 3 \mathrm{~b}$ ) located in the proximal tibia region also showed statistically differences between patient and control values for the whole set of MRI metrics. The only normal value was found for Tb.Th in ROI3a. More particularly, the differences between the patient and the controls were $44 \pm 18 \%$ for BVF, $-34 \pm 5 \%$ for Tb.Sp, $34 \pm 10 \%$ for Tb.N and $8 \pm 8 \%$ for Tb.Th. 
After 12 months of TNF treatment, the bone microstructure differences were reduced, although remaining statistically significant in most of the cases. For the BVF, the difference was reduced to $26 \pm 11 \%$ and remained statistically significant for ROI3 and ROI3b. The Tb.Th difference was also reduced to $3 \pm 6 \%$ thereby becoming not statistically significant for any of the three tibial ROIs. The Tb.Sp difference slightly decreased to $-28 \pm 7 \%$ but remained statistically significant $(p<0.01)$ for ROI3 and ROI3a but not for ROI3b. The Tb.N difference also decreased to $21 \pm 6 \%$ but remained statistically significant for ROI3 and ROI3a but not for ROI3b (table 2).

\section{Discussion}

In the present study, we assessed bone microarchitecture in a PA patient in order to document the potential bone quality changes associated with his inflammatory status. We also assessed the microarchitecture modification resulting from a one-year anti-TNF treatment. We mainly found that PET$\mathrm{FNa} / \mathrm{MRI}$ showed a largely inflamed knee articulation with some specific hypermetabolic regions in the vicinity of ligament and tendons in the patella, the distal femur, and the proximal tibia. Microarchitectural changes quantified using UHF MRI were affecting the whole bone segments and were not localized within the hypermetabolic regions only. After a year of TNF treatment, the combined PET-UHF MRI approach showed highly reduced hypermetabolic regions and an improvement for most of the microarchitectural parameters.

Before the treatment, all the microarchitecture metrics were significantly different with respect to the control values and so in at least one ROI. Using HR-pQCT of the distal radius, Kocijan et al. reported similar abnormalities with significantly reduced BVF and Tb.N in a group of PA patients [8]. Although previous DXA measurements have been controversial regarding BMD changes in PA patients [11]-[14], our results further support those obtained using a radiating imaging technique and confirm abnormalities of trabecular bone in PA patients so that osteoporotic changes might be expected in PA.

In the field of rheumatologic inflammatory disorders, our study is the first to address the bone microarchitecture issue using UHF MRI, although previous studies involving the use of UHF MRI have reported promising results in osteoporosis [20]-[22]. As an example, Chang et al. [20] found abnormal trabecular characteristics including BVF in the distal femur of subjects with fragility fractures whereas the DXA T-score was normal. Of interest, BVF, Tb.Sp and Tb.N were abnormal in the majority (7/8) of ROls in the present study whereas Tb.Th was abnormal in a limited number $(2 / 8)$ of ROIs. These results further support those previously reported by Kocijan et al [8] and Chang et al. [20] regarding the larger sensitivity of BVF, Tb.Sp and Tb.N to bone micro-architecture alterations as compared to Tb.Th. In fact, Kocijan et al. [8] reported no difference in Tb.Th between PA patients and healthy controls in distal radii while Chang et al. [20] found normal distal femur Tb.Th in patients with fragility fractures.

Trabecular abnormalities detected using UHF MRI were found in all the hypermetabolic regions detected using PET-FNa, showing that microarchitecture deterioration was affecting the whole bone segments. The PET analysis has been shown to reflect bone remodelling and has been used in several studies on 
osteoporosis [34]-[37]. In our case, PET-FNa allowed us to localize specific ROIs characterized by elevated hypermetabolic activity before treatment and ROIs presenting partial or full remission after treatment.

After a year of anti-TNF treatment, the trabecular parameters clearly illustrated that the knee of the patient was in clinical remission from his PA status. The trabecular parameters reversal might result from the decreased inflammatory status leading to a reduced osteoclastic bone resorption activity. In PA, Hoff et al. [16] have showed that 24 weeks of Infliximab treatment can stop the bone loss. In multiple studies conducted in rheumatoid arthritis (RA) patients, the TNF blocking strategy has been associated with an increase of biologicals markers indicating bone formation and a decrease of those illustrating bone resorption [38]-[40]. In both RA and Ankylosing spondylitis (AS), the efficiency of anti-TNF agents on bone loss has also been confirmed through BMD measurements using DXA $(39-41,42,43)$. Our PET$\mathrm{FNa} / \mathrm{MRI}$ measurements also supported the efficiency of the anti-TNF strategy. In fact, UHF MRI allowed us to assess and quantify the microarchitectural parameters in the hypermetabolic ROIs assessed through the PET-FNa. In our study, UHF MRI showed an almost homogeneous microarchitecture deterioration before treatment and a partial or a complete remission after one year of treatment. These results are also in agreement with those previously reported as a result of bisphosphonates treatment in osteoporotic patients [35], [37].

A few limitations have to be acknowledged in the present study. Although, this preliminary study was conducted in a PA patient we have quantified morphological parameters, of several UHF MR images, from 3 different bone segments (patella, distal femur, and proximal tibia) and using 8 different ROls. Moreover, the results of the PA patient were compared both temporally, i.e. before and after the treatment, and against the control group.

The investigation of bone microarchitecture in patients affected by PA is of interest for a reliable assessment of bone quality, illness risk stratification and for the follow-up therapeutic strategy. Up to now, PA patients have been mainly treated using CsDMARD, bDMARD and tsDMARD [46] and the improvements of bone microarchitecture have never been assessed. However, the administration of antiTNF may inhibit the osteoclastic action of bone resorption triggered by the inflammatory response. Moreover, the application of UHF MRI might be of high interest to explore bone microarchitecture in the future and could be applied to investigate some specific clinical situations.

\section{Declarations}

The present study complies with the Declaration of Helsinki, Aix Marseille University ethics committee has approved the research protocol and informed consent has been obtained from the subjects.

All authors have given consent to the publication of the following paper

The datasets analyzed during the current study are available from the corresponding author on reasonable request. 
All authors declare no conflict of interest.

ES has received funding from the European Union's Horizon 2020 research and innovation program under the Marie Skodowska-Curie grant agreement No713750. Also, it has been carried out with the financial support of the Regional Council of Provence-Alpes-Côte d'Azur and with the financial support of the $A^{*}$ MIDEX ( $n^{\circ}$ ANR- 11-IDEX-0001-02), funded by the "Investissements d'Avenir" project funded by the French Government, managed by the French National Research Agency (ANR).

SGu and DB concepted and designed the work. LE and SGu carefully recruited the patients. SGa and SC perfomerd the PET-FNa acquisition and JPM performed the UHF MRI acquisition. ES analyzed and interpreted the patient data. ES and LE wrote the first draft of the paper. All authors read and approved the final manuscript.

\section{References}

1. C. E. Griffiths et J. N. Barker, «Pathogenesis and clinical features of psoriasis », Lancet Lond. Engl., vol. 370, no 9583, p. 263-271, juill. 2007, doi: 10.1016/S0140-6736(07)61128-3.

2. J. M. Moll et V. Wright, « Psoriatic arthritis », Semin. Arthritis Rheum., vol. 3, n 1, p. 55-78, 1973, doi: 10.1016/0049-0172(73)90035-8.

3. A. Ogdie et P. Weiss, "The Epidemiology Psoriatic Arthritis ", Rheum. Dis. Clin. North Am., vol. 41, n 4, p. 545-568, nov. 2015, doi: 10.1016/j.rdc.2015.07.001.

4. A. P. Villani et al., «Prevalence of undiagnosed psoriatic arthritis among psoriasis patients: Systematic review and meta-analysis ", J. Am. Acad. Dermatol., vol. 73, nº 2, p. 242-248, août 2015, doi: 10.1016/j.jaad.2015.05.001.

5. C. T. Ritchlin, R. A. Colbert, et D. D. Gladman, «Psoriatic Arthritis », N. Engl. J. Med., vol. 376, nº 10, p. 957-970, mars 2017, doi: 10.1056/NEJMra1505557.

6. E. F. A. Leijten et al., « Brief report: enrichment of activated group 3 innate lymphoid cells in psoriatic arthritis synovial fluid ", Arthritis Rheumatol. Hoboken NJ, vol. 67, n 10 , p. 2673-2678, oct. 2015, doi: 10.1002/art.39261.

7. C. Ritchlin, S. A. Haas-Smith, D. Hicks, J. Cappuccio, C. K. Osterland, et R. J. Looney, « Patterns of cytokine production in psoriatic synovium », J. Rheumatol., vol. 25, nº 8, p. 1544-1552, août 1998.

8. R. Kocijan et al., "Quantitative and Qualitative Changes of Bone in Psoriasis and Psoriatic Arthritis Patients », J. Bone Miner. Res. Off. J. Am. Soc. Bone Miner. Res., vol. 30, nº 10, p. 1775-1783, oct. 2015, doi: 10.1002/jbmr.2521.

9. B. Menon et al., «Interleukin-17+CD8+ T cells are enriched in the joints of patients with psoriatic arthritis and correlate with disease activity and joint damage progression », Arthritis Rheumatol. Hoboken NJ, vol. 66, nº 5, p. 1272-1281, mai 2014, doi: 10.1002/art.38376.

10. C. T. Ritchlin, S. A. Haas-Smith, P. Li, D. G. Hicks, et E. M. Schwarz, « Mechanisms of TNF-alpha- and RANKL-mediated osteoclastogenesis and bone resorption in psoriatic arthritis », J. Clin. Invest., vol. 
$111, n^{\circ} 6$, p. 821-831, mars 2003, doi: 10.1172/JCl16069.

11. L. M. Perez-Chada et J. F. Merola, « Comorbidities associated with psoriatic arthritis: Review and update », Clin. Immunol. Orlando Fla, vol. 214, p. 108397, mai 2020, doi:

10.1016/j.clim.2020.108397.

12. A. Ogdie et al., "The risk of fracture among patients with psoriatic arthritis and psoriasis: a population-based study », Ann. Rheum. Dis., vol. 76, n 5, p. 882-885, mai 2017, doi: 10.1136/annrheumdis-2016-210441.

13. S. Chandran, A. Aldei, S. R. Johnson, A. M. Cheung, D. Salonen, et D. D. Gladman, «Prevalence and risk factors of low bone mineral density in psoriatic arthritis: A systematic review ", Semin. Arthritis Rheum., vol. 46, n² 2, p. 174-182, 2016, doi: 10.1016/j.semarthrit.2016.05.005.

14. A. Del Puente et al., "Fragility Fractures in Patients with Psoriatic Arthritis », J. Rheumatol. Suppl., vol. 93, p. 36-39, nov. 2015, doi: 10.3899/jrheum.150633.

15. O. Di Munno et F. Ferro, «The effect of biologic agents on bone homeostasis in chronic inflammatory rheumatic diseases », Clin. Exp. Rheumatol., vol. 37, n 3, p. 502-507, juin 2019.

16. M. Hoff, A. Kavanaugh, et G. Haugeberg, « Hand bone loss in patients with psoriatic arthritis: posthoc analysis of IMPACT II data comparing infliximab and placebo », J. Rheumatol., vol. 40, n 8, p. 1344-1348, août 2013, doi: 10.3899/jrheum.121376.

17. N. Maruotti, A. Corrado, et F. P. Cantatore, "Osteoporosis and rheumatic diseases », Reumatismo, vol. 66 , $n^{\circ} 2$, p. 125-135, juin 2014, doi: 10.4081/reumatismo.2014.785.

18. K. Briot et al., « 2018 update of French recommendations on the management of postmenopausal osteoporosis », Joint Bone Spine, vol. 85, n 5, p. 519-530, oct. 2018, doi: 10.1016/j.jbspin.2018.02.009.

19. G. Chang et al., « MRI Assessment of Bone Structure and Microarchitecture », J. Magn. Reson. Imaging JMRI, vol. 46, n 2, p. 323-337, août 2017, doi: 10.1002/jmri.25647.

20. G. Chang et al., « 7 Tesla MRI of bone microarchitecture discriminates between women without and with fragility fractures who do not differ by bone mineral density ", J. Bone Miner. Metab., vol. 33, $\mathrm{n}^{\circ}$ 3, p. 285-293, mai 2015, doi: 10.1007/s00774-014-0588-4.

21. D. Guenoun et al., «Correlative Analysis of Vertebral Trabecular Bone Microarchitecture and Mechanical Properties: A Combined Ultra-high Field (7 Tesla) MRI and Biomechanical Investigation », Spine, vol. 42, n² 20, p. E1165-E1172, oct. 2017, doi: 10.1097/BRS.0000000000002163.

22. D. Guenoun et al., « Assessment of proximal femur microarchitecture using ultra-high field MRI at 7 Tesla », Diagn. Interv. Imaging, vol. 101, nº 1, p. 45-53, janv. 2020, doi: 10.1016/j.diii.2019.06.013.

23. M. de Arcocha, H. Portilla-Quattrociocchi, P. Medina-Quiroz, et J. M. Carril, « Estado actual del uso del (18F)fluoruro sódico en la patología ósea », Rev. Esp. Med. Nucl. E Imagen Mol., vol. 31, n 1, p. 51-57, janv. 2012, doi: 10.1016/j.remn.2011.05.008. 
24. M. Jenkinson, P. Bannister, M. Brady, et S. Smith, « Improved Optimization for the Robust and Accurate Linear Registration and Motion Correction of Brain Images », Neurolmage, vol. 17, $n^{\circ} 2$, p. 825-841, oct. 2002, doi: 10.1006/nimg.2002.1132.

25. K. Makki, B. Borotikar, M. Garetier, S. Brochard, D. Ben Salem, et F. Rousseau, « In vivo ankle joint kinematics from dynamic magnetic resonance imaging using a registration-based framework », J. Biomech., vol. 86, p. 193-203, mars 2019, doi: 10.1016/j.jbiomech.2019.02.007.

26. V. Arsigny, O. Commowick, N. Ayache, et X. Pennec, « A Fast and Log-Euclidean Polyaffine Framework for Locally Linear Registration », J. Math. Imaging Vis., vol. 33, n² 2, p. 222-238, févr. 2009, doi: 10.1007/s10851-008-0135-9.

27. S. Cammilleri et al., " Knee psoriatic enthesitis assessed using positron emission tomography (PET) FNa merged to ultrahigh field magnetic resonance imaging (UHF-MRI) », Joint Bone Spine, vol. 86, $\mathrm{n}^{\circ}$ 3, p. 387-388, 2019, doi: 10.1016/j.jbspin.2019.01.002.

28. G. Chang et al., « 3 Tesla MRI detects deterioration in proximal femur microarchitecture and strength in long-term glucocorticoid users compared with controls: Changes in Proximal Femur Microarchitecture in GIO », J. Magn. Reson. Imaging, vol. 42, nº 6, p. 1489-1496, déc. 2015, doi: 10.1002/jmri.24927.

29. C. S. Rajapakse, M. B. Leonard, Y. A. Bhagat, W. Sun, J. F. Magland, et F. W. Wehrli, « Micro-MR Imaging-based Computational Biomechanics Demonstrates Reduction in Cortical and Trabecular Bone Strength after Renal Transplantation », Radiology, vol. 262, n 3, p. 912-920, mars 2012, doi: 10.1148/radiol.11111044.

30. P. Getreuer, « Chan-Vese Segmentation », Image Process. Line, vol. 2, p. 214-224, août 2012, doi: 10.5201/ipol.2012.g-cv.

31. R. Dougherty et K.-H. Kunzelmann, " Computing Local Thickness of 3D Structures with ImageJ », Microsc. Microanal., vol. 13, nº S02, août 2007, doi: 10.1017/S1431927607074430.

32. E. Brun, C. Ferrero, et J. Vicente, « Fast Granulometry Operator for the 3D Identification of Cell Structures », Fundam. Informaticae, vol. 155, n 4, p. 363-372, sept. 2017, doi: 10.3233/FI-2017-1590.

33. D. Rosen, B. Herrington, P. Bhargava, R. Laucirica, et G. Verstovsek, « Correlation of Tissue Biopsy and Fine Needle Aspiration Cytology with Positron Emission Tomography Results », Pathol. Res. Int., vol. 2011, p. 1-7, avr. 2011, doi: 10.4061/2011/323051.

34. G. M. Blake, S.-J. Park-Holohan, et I. Fogelman, « Quantitative Studies of Bone in Postmenopausal Women Using 18F-Fluoride and 99mTc-Methylene Diphosphonate », J. Nucl. Med., vol. 43, nº 3, p. 338-345, janv. 2002.

35. M. L. Frost, G. J. R. Cook, G. M. Blake, P. K. Marsden, N. A. Benatar, et I. Fogelman, « A prospective study of risedronate on regional bone metabolism and blood flow at the lumbar spine measured by 18F-fluoride positron emission tomography ", J. Bone Miner. Res. Off. J. Am. Soc. Bone Miner. Res., vol. 18, nº 12, p. 2215-2222, déc. 2003, doi: 10.1359/jbmr.2003.18.12.2215. 
36. W. Raynor et al., " Assessing global uptake of 18F-sodium fluoride in the femoral neck: a novel quantitative technique to evaluate changes in bone turnover with age. », J. Nucl. Med., vol. $58, \mathrm{n}^{\circ}$ supplement 1, p. 1223-1223, janv. 2017.

37. K. Uchida et al., «Effects of alendronate on bone metabolism in glucocorticoid-induced osteoporosis measured by $18 \mathrm{~F}$-fluoride PET: a prospective study », J. Nucl. Med. Off. Publ. Soc. Nucl. Med., vol. 50, n ${ }^{0} 11$, p. 1808-1814, nov. 2009, doi: 10.2967/jnumed.109.062570.

38. M. Vis et al., « Early changes in bone metabolism in rheumatoid arthritis patients treated with infliximab », Arthritis Rheum., vol. 48, nº 10, p. 2996-2997, 2003, doi: 10.1002/art.11292.

39. M. Vis et al., "Evaluation of bone mineral density, bone metabolism, osteoprotegerin and receptor activator of the NFkappaB ligand serum levels during treatment with infliximab in patients with rheumatoid arthritis », Ann. Rheum. Dis., vol. 65, n 11, p. 1495-1499, nov. 2006, doi: 10.1136/ard.2005.044198.

40. U. Lange, J. Teichmann, U. Müller-Ladner, et J. Strunk, « Increase in bone mineral density of patients with rheumatoid arthritis treated with anti-TNF-alpha antibody: a prospective open-label pilot study », Rheumatol. Oxf. Engl., vol. 44, nº 12, p. 1546-1548, déc. 2005, doi: 10.1093/rheumatology/kei082.

41. H. Marotte, B. Pallot-Prades, L. Grange, P. Gaudin, C. Alexandre, et P. Miossec, « A 1-year case-control study in patients with rheumatoid arthritis indicates prevention of loss of bone mineral density in both responders and nonresponders to infliximab», Arthritis Res. Ther., vol. 9, n 3, p. R61, juin 2007, doi: 10.1186/ar2219.

42. C. A. Wijbrandts, R. Klaasen, M. G. W. Dijkgraaf, D. M. Gerlag, B. L. F. van Eck-Smit, et P. P. Tak, « Bone mineral density in rheumatoid arthritis patients 1 year after adalimumab therapy: arrest of bone loss », Ann. Rheum. Dis., vol. 68, n³ 3, p. 373-376, mars 2009, doi: 10.1136/ard.2008.091611.

43. M. Güler-Yüksel et al., " Changes in bone mineral density in patients with recent onset, active rheumatoid arthritis », Ann. Rheum. Dis., vol. 67, nº 6, p. 823-828, juin 2008, doi: 10.1136/ard.2007.073817.

44. F. Allali, M. Breban, R. Porcher, J. F. Maillefert, M. Dougados, et C. Roux, « Increase in bone mineral density of patients with spondyloarthropathy treated with anti-tumour necrosis factor alpha ", Ann. Rheum. Dis., vol. 62, nº 4, p. 347-349, avr. 2003, doi: 10.1136/ard.62.4.347.

45. S. Visvanathan et al., "Effects of infliximab on markers of inflammation and bone turnover and associations with bone mineral density in patients with ankylosing spondylitis ", Ann. Rheum. Dis., vol. 68, n² 2, p. 175-182, févr. 2009, doi: 10.1136/ard.2007.084426.

46. L. Gossec et al., «EULAR recommendations for the management of psoriatic arthritis with pharmacological therapies: 2019 update », Ann. Rheum. Dis., vol. 79, nº 6, p. 700-712, juin 2020, doi: 10.1136/annrheumdis-2020-217159.

\section{Tables}


Due to technical limitations, table xlsx are only available as a download in the Supplemental Files section.

\section{Figures}

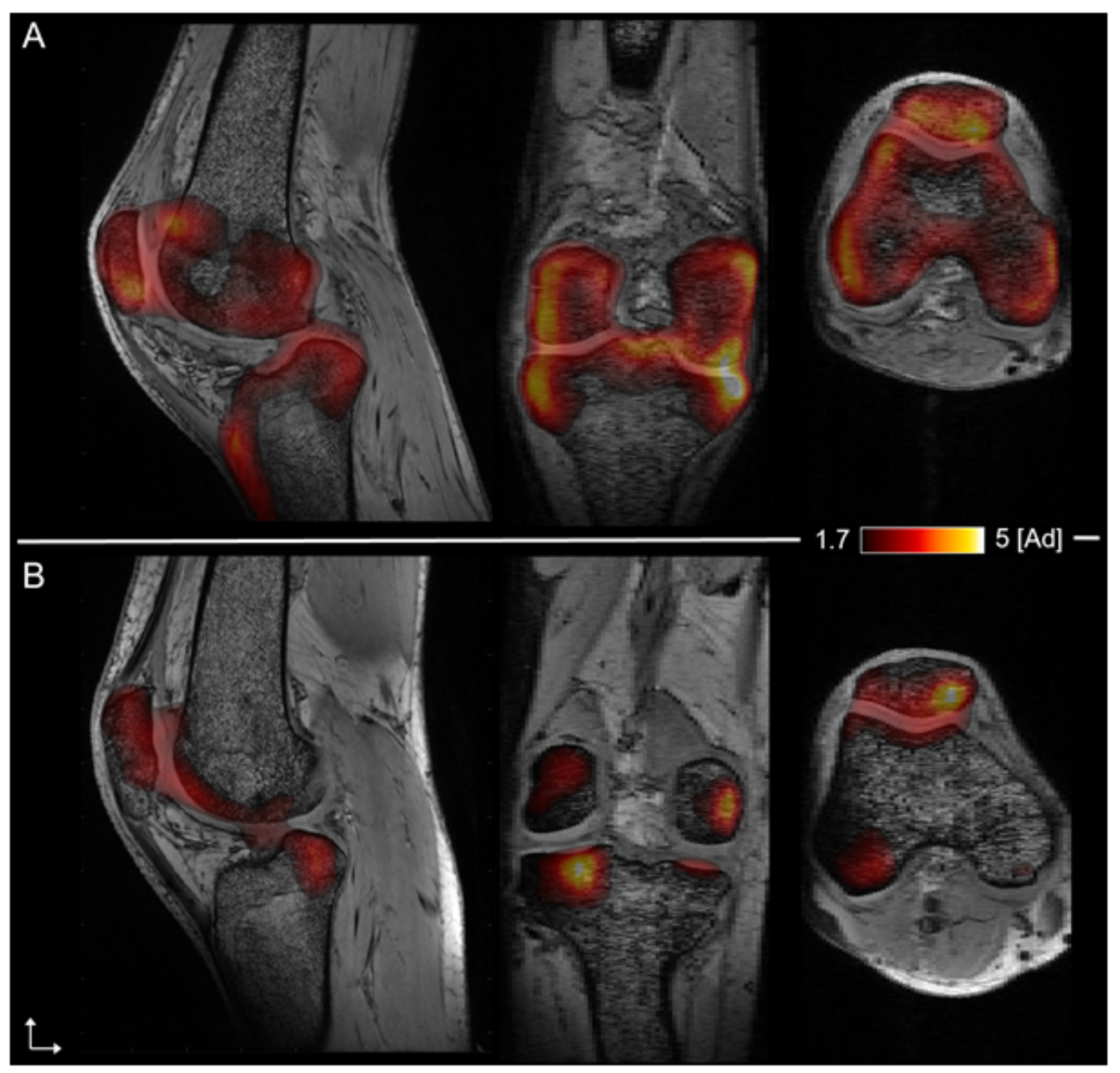

Figure 1

large hyperintensities were observed in the knee. The second PET image recorded after one year of treatment 


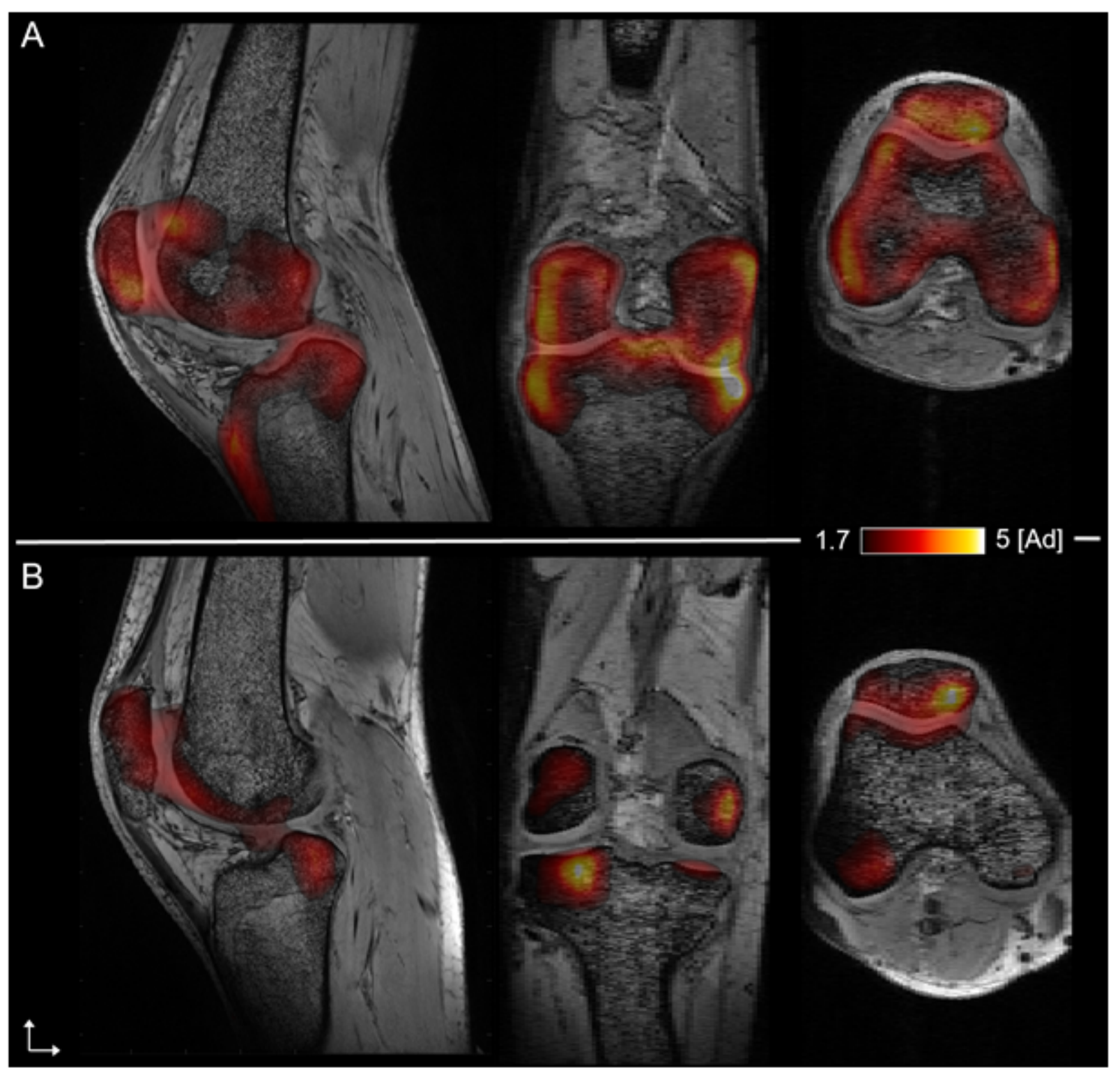

Figure 1

large hyperintensities were observed in the knee. The second PET image recorded after one year of treatment 


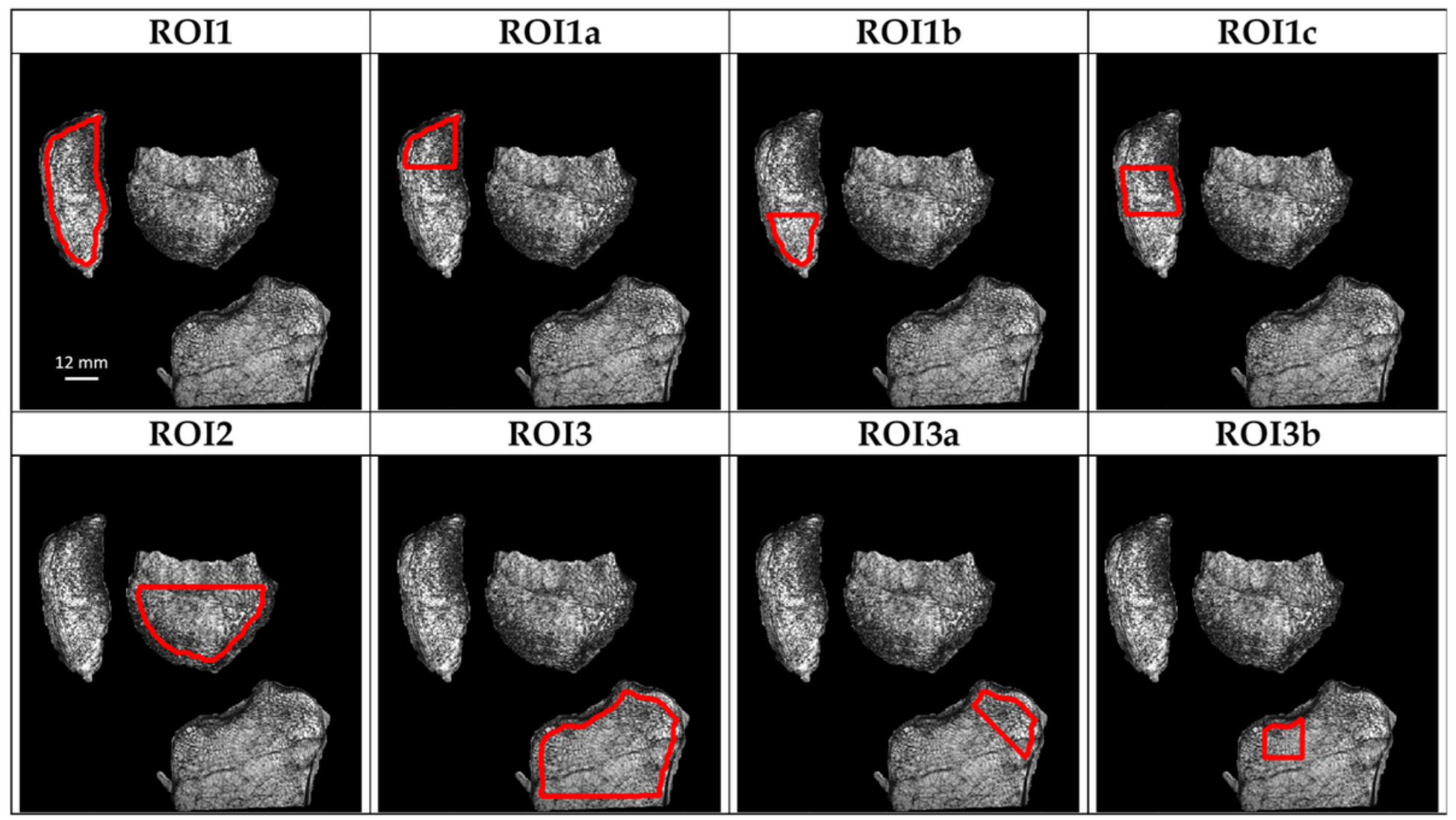

Figure 2

The corresponding filled contours were used as masks on which a 10-pixels closing process was applied $(2.34 \mathrm{~mm})$ in all directions in order to eliminate the cortical bone

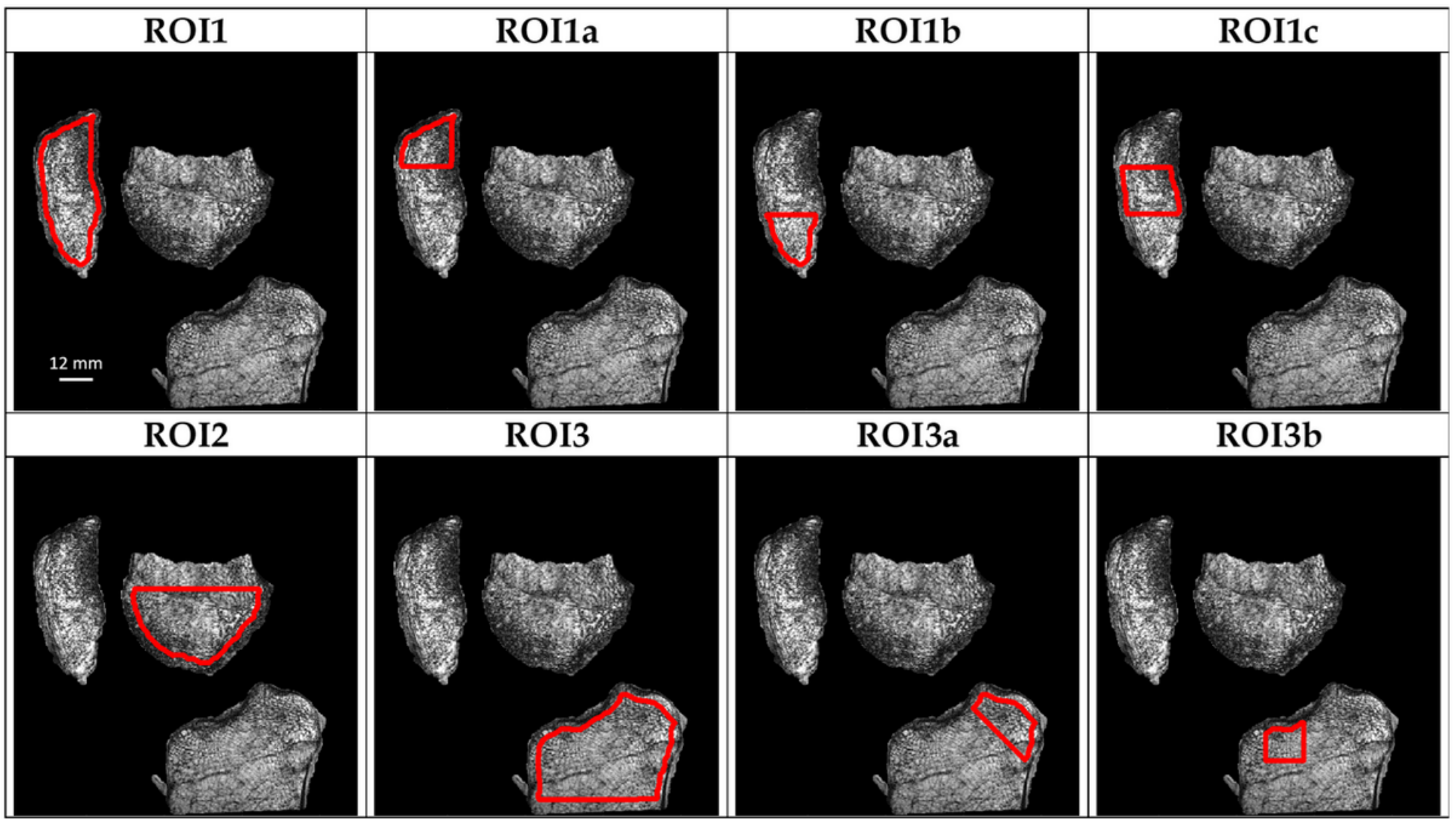


Figure 2

The corresponding filled contours were used as masks on which a 10-pixels closing process was applied $(2.34 \mathrm{~mm})$ in all directions in order to eliminate the cortical bone

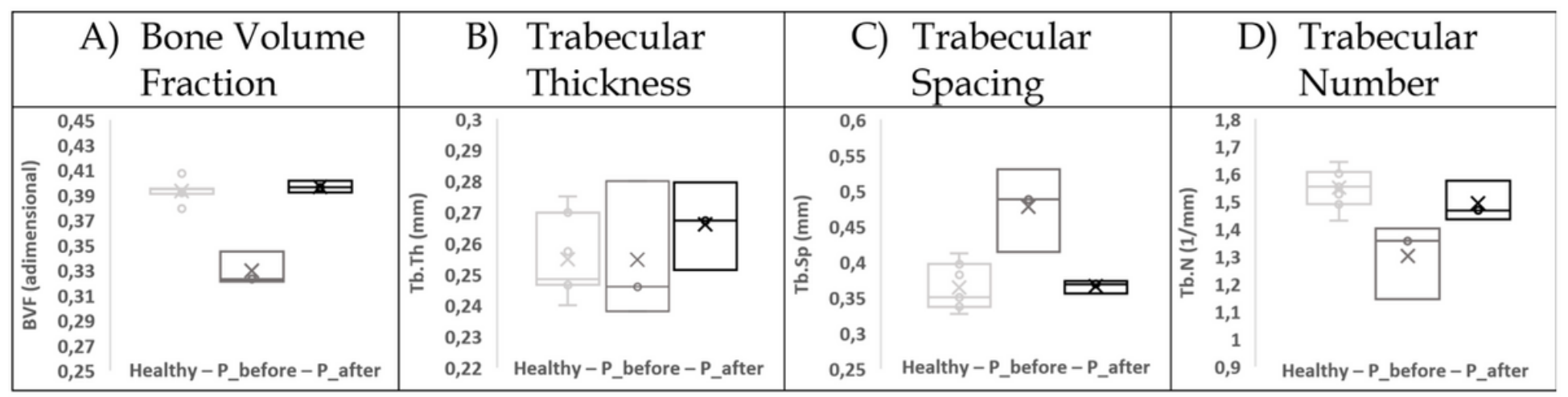

\section{Figure 3}

On the contrary, after the treatment, Tb.Th became significantly larger with a significant difference (up to 8\%) with controls and so for ROI1, ROI1b and ROI1c

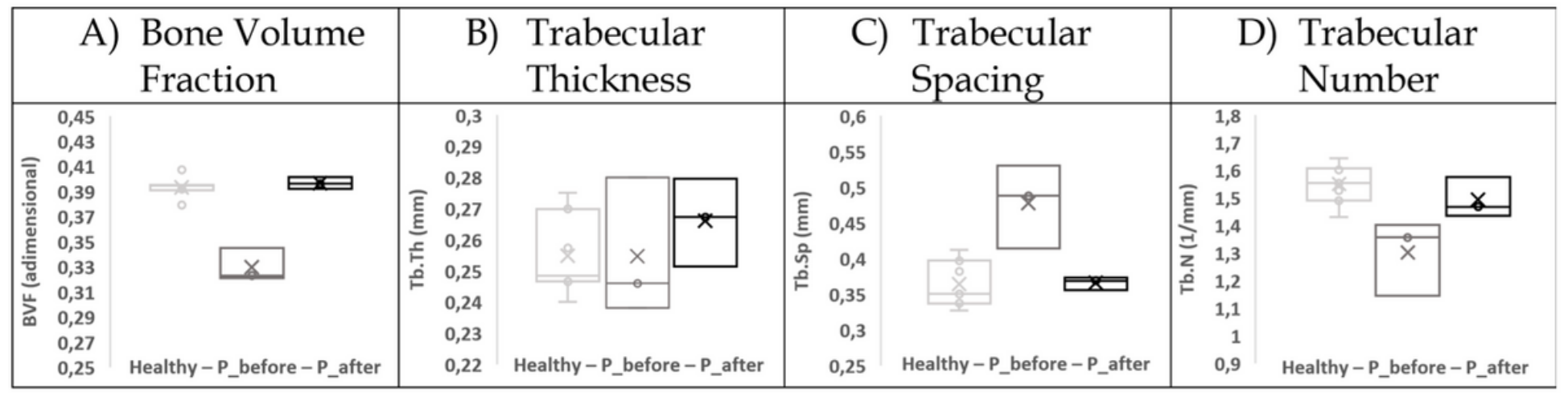

\section{Figure 3}

On the contrary, after the treatment, Tb.Th became significantly larger with a significant difference (up to $8 \%$ ) with controls and so for ROI1, ROI1b and ROI1c

\section{Supplementary Files}

This is a list of supplementary files associated with this preprint. Click to download.

- table1.xIsx

- table1.xlsx

- table2.xIsx

- table2.xlsx 